\title{
Open repair of chronic type B aortic dissection
}

\author{
Joel S. Corvera, Philip J. Hess Jr, John W. Fehrenbacher
}

Department of Surgery, Division of Cardiothoracic Surgery, Indiana University School of Medicine, Indianapolis, IN, USA

Contributions: (I) Conception and design: JS Corvera, PJ Hess Jr; (II) Administrative support: None; (III) Provision of study materials or patients: JS Corvera, JW Fehrenbacher; (IV) Collection and assembly of data: JS Corvera, JW Fehrenbacher; (V) Data analysis and interpretation: All authors; (VI) Manuscript writing: All authors; (VII) Final approval of manuscript: All authors.

Correspondence to: Joel S. Corvera, MD. Associate Professor of Clinical Surgery, Department of Surgery, Division of Cardiothoracic Surgery, Indiana University School of Medicine, 1801 N. Senate Boulevard, Suite 3300, Indianapolis, IN 46202, USA. Email: jcorvera@iuhealth.org.

\begin{abstract}
Open repair of descending thoracic and thoracoabdominal aortic aneurysms in the setting of chronic aortic dissection is a complex procedure that requires a thorough understanding of the physiology of aortic dissection and cardiopulmonary bypass, efficient reperfusion of vital organs and meticulous surgical technique. Open surgery on the descending thoracic or thoracoabdominal aorta is high risk and has been associated with significant morbidity and mortality. Alternatively, endovascular repair of chronic distal aortic dissection using the currently available thoracic endovascular devices is less invasive and can have low mortality. However, reintervention is quite common, the durability of the repair is questionable and aortic remodeling is uncommon in the abdominal aorta. Endovascular repair is not likely to be durable in the young patient with a genetically triggered or familial aortopathy. For these reasons, we believe that open repair of chronic distal aortic dissection is the definitive and durable solution in the appropriately selected patient. We have a greater than 20-year experience using deep hypothermia and circulatory arrest for repair of complex thoracic and thoracoabdominal aortic aneurysms. Morbidity and mortality in our hands using this perfusion technique in chronic distal aortic dissection is comparable to endovascular procedures with rates of mortality $3.6 \%$, permanent neurological deficit $2.6 \%$, permanent requirement for hemodialysis $0 \%$ and cerebrovascular accident $1 \%$. The following article describes, in detail, the intraoperative technique and postoperative management of patients who are suitable for descending thoracic or thoracoabdominal aortic aneurysm repair of chronic distal aortic dissection with deep hypothermia and circulatory arrest.
\end{abstract}

Keywords: Chronic aortic dissection; open thoracoabdominal aortic aneurysm repair; hypothermia

Received: 11 April 2020; Accepted: 22 May 2020; Published: 20 October 2021.

doi: 10.21037/jovs-20-99

View this article at: http://dx.doi.org/10.21037/jovs-20-99

\section{Introduction}

Open repair of descending thoracic and thoracoabdominal aortic aneurysms in the setting of chronic aortic dissection is a complex procedure that requires a thorough understanding of the physiology of aortic dissection and cardiopulmonary bypass, efficient reperfusion of vital organs and meticulous surgical technique. Open surgery on the descending thoracic or thoracoabdominal aorta is high risk and has been associated with significant morbidity and mortality (1-5). Alternatively, endovascular repair of chronic distal aortic dissection using the currently available thoracic endovascular devices is less invasive and can have low mortality. However, reintervention is quite common, the durability of the repair is questionable and aortic remodeling is uncommon in the abdominal aorta (6-9). Endovascular repair is not likely to be durable in the young patient with a genetically triggered or familial aortopathy. For these reasons, we believe that open repair of chronic distal aortic dissection is the definitive and durable solution in the appropriately selected patient.

We have a greater than 20-year experience using deep 
hypothermia and circulatory arrest for repair of complex thoracic and thoracoabdominal aortic aneurysms (10-12). Morbidity and mortality in our hands using this perfusion technique in chronic distal aortic dissection is comparable to endovascular procedures with rates of mortality $3.6 \%$, permanent neurological deficit $2.6 \%$, permanent requirement for hemodialysis $0 \%$ and cerebrovascular accident $1 \%$ (10). The following article describes, in detail, the intraoperative technique and postoperative management of patients who are suitable for descending thoracic or thoracoabdominal aortic aneurysm repair of chronic distal aortic dissection with deep hypothermia and circulatory arrest.

\section{Indications and patient selection}

Elfeteriades and colleagues have published elegant studies discussing the size at which thoracic aneurysms merit operative repair. In general, patients with asymptomatic descending thoracic aneurysms $6 \mathrm{~cm}$ in diameter or larger should be evaluated for repair due to the high risk of aortic complications $(13,14)$. However, recent data from Kim and colleagues suggest that repair at a smaller diameter is indicated as the aortic event risk of an unrepaired descending or thoracoabdominal aneurysm is $5.5-8.0 \%$ with an aortic diameter of $5.0 \mathrm{~cm}$ and $7.2-11.2 \%$ with an aortic diameter of $5.5 \mathrm{~cm}(15)$. Aneurysms with rapid growth (greater than $0.5 \mathrm{~cm} /$ year) or symptomatic aneurysms with chronic dissection are repaired at diameters less than 5.5 $\mathrm{cm}(10,12,15)$. As a general guideline, we have repaired descending thoracic and thoracoabdominal aneurysms as a result of a chronic aortic dissection at a diameter of 5.5 $\mathrm{cm}$. In patients with a genetically-triggered or familial aortopathy, open repair is initiated at $5.0 \mathrm{~cm}$. Although, seldom discussed, it is critical not to underestimate the true size of the aortic diameter due to the oval shape of a chronic aortic dissection. Three dimensional multiplanar reconstruction and center-line reconstruction of the images facilitates accurate measurement.

Patient selection is a subjective decision based on patient factors, procedural factors and surgeon experience. Although we do not routinely calculate frailty scores to guide the decision to operate, patients must be robust in that they should be able to ambulate independently, not have significant neurocognitive deficits, and be less than 85 years of age. Chronic pulmonary disease and the use of supplemental oxygen is not necessarily a contraindication for surgery. Chronic bronchitis and bronchiectasis with productive cough often complicate the postoperative course. Poor left ventricular function is not an absolute contraindication, however may require the prolonged use of inotropic and vasopressor agents postoperatively. Nonrevascularizable, severe multivessel coronary artery disease is a contraindication to hypothermic fibrillatory arrest that is required for this technique of thoracoabdominal aneurysm repair. In the surgeon's eye, the patient must be able to tolerate a significantly large incision and a lengthy perfusion time.

\section{Preoperative evaluation and testing}

A computed tomographic angiogram (CTA) with intravenous contrast of the chest, abdomen, and pelvis is used to estimate the diameter of the thoracoabdominal aorta, assess the extent of the aneurysm and dissection, interrogate the origins of the visceral arteries and iliofemoral arteries from either the true or false lumen and assess alternative arterial cannulation sites. Coronary catheterization is performed on all elective and urgent patients when possible, as up to $20 \%$ of patients require preoperative or intraoperative coronary revascularization (11). Significant coronary stenosis should be revascularized preoperatively or intraoperatively since the technique requires a duration of hypothermic fibrillation. Transthoracic echocardiogram evaluates for significant aortic valve insufficiency and other significant valvular heart disease. If $3+$ or greater aortic insufficiency exists, it must be repaired as a first stage operation prior to the distal repair or is relative contraindication using deep hypothermia and circulatory arrest.

\section{Anesthetic technique and monitoring}

Anesthetic technique is composed of total intravenous anesthesia using propofol and remifentanil to allow preservation of motor-evoked and somatosensory-evoked potentials for spinal cord monitoring. Placement of a double-lumen endotracheal tube or single lumen tube with bronchial blocker is used for selective airway control. Confirmation of proper positioning with fiber optic bronchoscopy is essential to achieve single lung ventilation. A transfusion system with fluid warming capabilities (Level 1 H-1200 Fast Flow Fluid Warmer, Smiths Medical, Dublin, $\mathrm{OH}$ ) is useful for administration of blood or blood products after separation from cardiopulmonary bypass.

A right radial arterial line is preferred as the left 


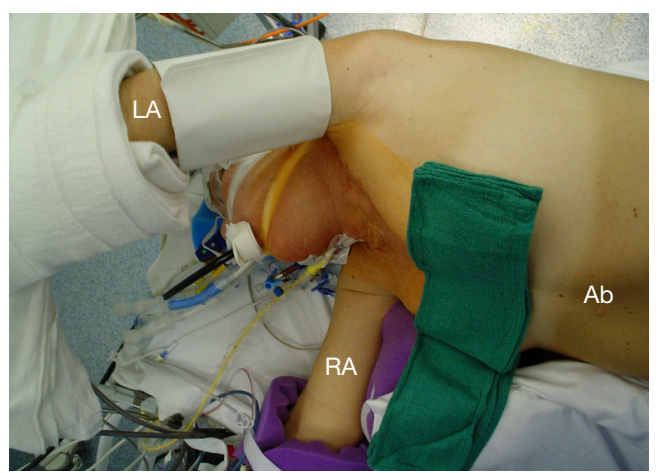

Figure 1 Positioning of patient with the left arm overhead for replacement of the proximal descending aorta with extension into the aortic arch. LA, left arm; RA, right arm; Ab, abdomen.

subclavian artery may need to be temporarily occluded during the operation. A pulmonary artery catheter is used for hemodynamic evaluation as most patients require temporary inotropic support in the early postoperative period. Transcranial infrared oxygen sensors are placed on the left and right forehead (INVOS Cerebral/Somatic Oximeter, Somentics Corporation, Troy, MI). Motorevoked and somatosensory-evoked potentials (Cadwell Cascade stimulator, detector, disposable subdermal needle electrodes, Cadwell Laboratories, Inc., Kennewick, WA) are recorded after induction of anesthesia for baseline values and are assessed intraoperatively after separation from cardiopulmonary bypass and after femoral arterial and venous cannulas have been removed. Transesophageal echocardiography (TEE) is used to assure proper guidewire placement in the right atrium prior to passage of the femoral venous cannula. TEE is also used to reassess degree of aortic insufficiency on cardiopulmonary bypass, which may dictate the immediate placement of a left ventricular decompression catheter (Argyle Left Ventricular Sump Vent Catheter, Covidien, Mansfield, MA) once the heart fibrillates during the cooling period. In addition, TEE or epiaortic ultrasound scanning may be used to evaluate the descending thoracic aorta for intraluminal debris to guide in the occasional need for direct aortic cannulation. Bladder or rectal temperatures as well as tympanic or nasopharyngeal temperatures are monitored.

Methylprednisolone sodium succinate (1 gram) is given intravenously prior to skin incision. Aminocaproic acid $(10 \mathrm{mg})$ is administered intravenously at the start of the procedure to reduce fibrinolytic coagulopathy and is readministered $(5 \mathrm{mg})$ if the cardiopulmonary bypass time exceeds 3 hours.

Lumbar drains are routinely placed preoperatively for Crawford extent II and III thoracoabdominal aneurysm repairs. In our experience, these extensive procedures carry the greatest risk of spinal cord ischemia (10-12). We have not routinely placed lumbar drains for extent I and IV thoracoabdominal aneurysm repairs, as we have experienced few instances of spinal cord ischemia using deep hypothermia. Reasons for placing a lumbar drain in extent I or IV thoracoabdominal aneurysm repair can include prior open or endovascular distal aortic surgery.

\section{Positioning and incisions}

The patient is positioned 90 -degree right lateral decubitus at the shoulders with the hips rotated to 45 degrees for bilateral groin access for femoral artery and vein cannulation. Placing the left arm overhead is imperative when exposure of the transverse arch or proximal aortic cannulation is anticipated (Figure 1).

Incisions vary and are shown according to aneurysmal extents in (Figure 2). The thoracic portion of the incision to expose the entire descending thoracic aorta and Crawford I or II extent thoracoabdominal aneurysms begins on the posterolateral chest entering the fifth intercostal space and transitioning to the sixth intercostal space at the anterior axillary line. Transection of the costal margin of the seventh rib is necessary for extensive descending thoracic or thoracoabdominal aneurysms. Entry into the chest can be lower for Crawford extent III or IV (seventh or eighth intercostal space) thoracoabdominal aneurysm repairs. Entry into the thoracic cavity at intercostal spaces lower than the eighth limits access to the left ventricular apex for decompression and to the left inferior pulmonary vein for additional venous drainage if needed.

The diaphragmatic hiatus is enlarged posteriorly for extensive descending thoracic aneurysms or Crawford extent I thoracoabdominal aneurysms. The celiac artery can be exposed without significant division of the diaphragm. The diaphragm is divided circumferentially for all other thoracoabdominal aneurysms. The incision on the abdominal wall is placed lateral to the border of the left rectus muscle and extends caudally to an imaginary horizontal line drawn at the level of the umbilicus. The preperitoneal and retroperitoneal spaces are developed with blunt dissection, cauterizing small vessels that traverse the plane of dissection. Entry into the peritoneal space can be generally avoided. 


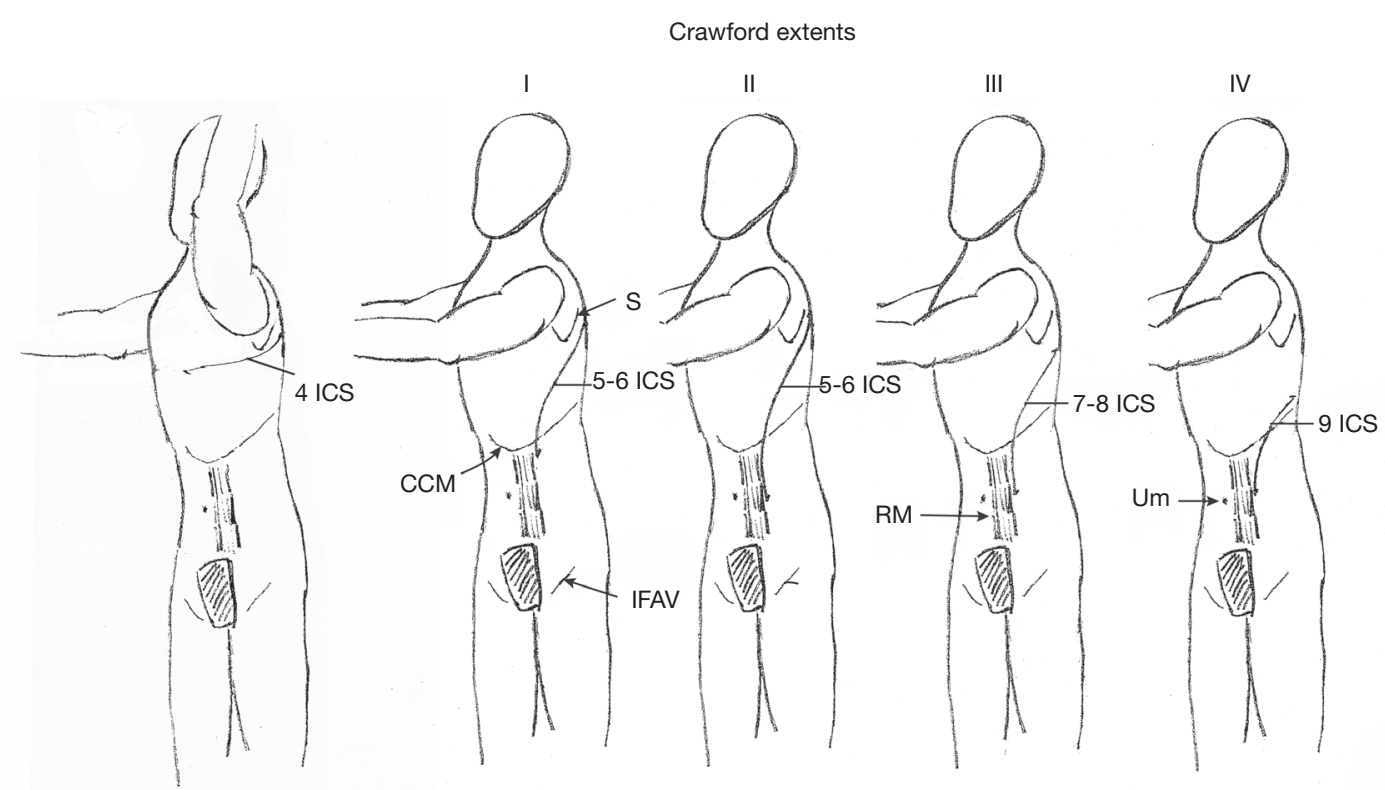

Figure 2 Patient position and incision for aneurysm repair of the proximal descending thoracic aorta and Crawford Extents I-IV. C I, II, III, IV, Crawford extent aneurysms; ICS, intercostal space; S, scapula; RM, rectus muscle; CCM, costochondral margin; IFAV, incision over femoral artery vein; Um, umbilicus.

\section{Perfusion set up and technique}

The cardiopulmonary bypass circuit for thoracoabdominal aneurysm repair with deep hypothermia and circulatory arrest at our institution has evolved into a complex system that allows regulation of flow for three separate arterial outflows. The main arterial circuit has two lines, one of which traverses two roller heads to allow differential flow through the two lines. Essentially, the second arterial line (which goes through two roller heads) "steals" flow from the main arterial line. A third arterial circuit runs blood through the cardioplegia line without additive (Quest Myocardial Protection System, Quest Medical, Inc, Allen, TX) and is connected to a multiple perfusion set (DLP Multiple Perfusion Set, Medtronic, Inc., Minneapolis, $\mathrm{MN}$ ). It is used to provide cold blood perfusion to the celiac artery, superior mesenteric artery and bilateral renal arteries through direct cannulation with balloon tipped perfusion catheters (9F Pruitt Irrigation Occlusion Catheter, LeMaitre Vascular, Inc. Burlington, MA).

When the patient is placed on cardiopulmonary bypass and systemic cooling initiated, mean arterial pressures of 40-50 mmHg are acceptable. Maintaining a cardiac index of $2.2 \mathrm{~L} / \mathrm{m}^{2}$ is not always possible while cooling, however a radial artery pressure of $40 \mathrm{mmHg}$ or higher is recommended. Insertion of a second arterial or venous cannula may be indicated to achieve these results. During the warming phase and as normothermic temperatures are being reached, mean arterial pressure of $70-90 \mathrm{mmHg}$ should be targeted as pump flows are increased to maintain full flow based on body surface area calculation.

\section{Surgical technique}

\section{Descending thoracic aneurysm repair}

The chest is opened and a self-retaining retractor (OmniTract Surgical Retractor, Omni-Tract Surgical, St. Paul, $\mathrm{MN}$ ) is placed for adequate exposure. The aorta is exposed and the patient is administered full pump dose heparin, 300 units $/ \mathrm{kg}$. Venous cannulation utilizes a long dualstage 24/29 Fr. or 30/33 Fr. cannula (Carpentier Bi-Caval Femoral Venous Cannula, Medtronic, Inc., Minneapolis, $\mathrm{MN}$ ) that is passed over a guidewire and directed into the right atrium with TEE guidance. It is extremely rare to need fluoroscopy to assist in the placement of the guidewire and cannula in the right atrium. If the venous line cannot be passed safely usually to iliac vein stenosis or compression, the pulmonary artery or the left atrium through the left atrial appendage or left inferior pulmonary vein can be 
directly cannulated with a 36 or 38 Fr. single stage cannula (DLP Single Stage Venous Cannula, Medtronic, Inc., Minneapolis, $\mathrm{MN}$ ) for venous drainage.

Cannulation of the femoral artery using the Seldinger technique with a 20-Fr. cannula (Fem-flex II Femoral Arterial Cannula, Edwards Lifesciences, Irvine, CA) is used in the majority of cases although stepping down to a 16 or 18 -Fr. cannula in smaller vessel diameters may be appropriate in smaller patients. Occasionally, the common femoral artery is very small requiring suturing of an $8 \mathrm{~mm}$ Dacron graft (Vascutek Gelsoft Straight Graft, Terumo Cardiovascular Corporation, Renfrewshire, Scotland, UK) to the femoral artery. In patients with severe atherosclerotic disease of the abdominal and/or thoracic aorta, the proximal descending aorta or distal transverse arch can be directly cannulated placing the proximal end of the cannula in non-diseased, non-dissected arch or ascending aorta. An arterial cannula using a guidewire and transesophageal echo guidance is especially helpful in these circumstances. More proximal aortic cannulation will avoid retrograde flow in a diseased thoracoabdominal aorta and may prevent retrograde embolization to the cerebral circulation. Arterial blood is cooled to 15 degrees Centigrade and the cooling period is for a minimum of 30 minutes.

During cooling, the pericardium is opened over the left ventricular apex. Immediately after the heart fibrillates, a 12 Fr. left ventricular decompression catheter (Argyle Left Ventricular Sump Vent Catheter, Covidien, Mansfield, MA) is inserted through a stab incision in the left ventricular apex. Left ventricular decompression is especially important if any aortic insufficiency exists in order to avoid pulmonary venous congestion in the lungs. Pulmonary venous congestion along with left lung manipulation during systemic anticoagulation can cause troublesome intraparenchymal bleeding in the left lung. Avoiding pulmonary artery pressures greater than $15 \mathrm{mmHg}$ through left ventricular decompression and very gentle traction on and manipulation of the left lung are critical in minimizing this complication.

The proximal extent of the aneurysm is exposed. Isolation of the arch proximal to the aneurysm is not always necessary since aortic clamps are not necessary. Intercostal arteries of the proximal half of the descending thoracic aorta are ligated and divided on the outside of the aorta minimizing the manipulation of the aneurysm. Ligation of these intercostal arteries on the outside of the aorta prior to opening the aneurysm may prevent a steal phenomenon of spinal cord blood flow $(16,17)$. Intercostal arteries in the distal half of the descending thoracic aorta are not ligated, not only to allow cold blood perfusion of the distal cord, but to preserve them to be reimplanted later in the procedure. A small polyethylene tube (Intramedic Polyethylene Tubing, Becton, Dickinson and Company, Franklin Lakes, $\mathrm{NJ}$ ) is passed around the mid descending thoracic aorta, staying close to the aortic wall to avoid damaging the large lymphatic vessels as well as the hemiazygos vein. This tube will mark the site for placement of cross-clamp during formation of the proximal anastomosis. Encircling the aneurysm should be performed at a place where the aneurysm narrows, if possible.

Once hypothermia has been achieved (a minimum of 30 minutes of cooling and up to 45 minutes of cooling when a complex proximal anastomosis is anticipated), the patient is placed in a steep Trendelenburg position and rotated to the patient's right to place the ascending aorta in the most dependent position. Positioning in this manner will help keep air out of the aortic arch branches. Potassium chloride, 40-80 milliequivalents, is added directly into the cardiopulmonary bypass circuit which causes a temporary cardiac diastolic arrest. Cardiopulmonary bypass is stopped and the LV suction is halted. If suction on the $\mathrm{LV}$ decompression is continued, it will entrain air into the transverse arch, ascending aorta and left ventricle. The venous line is clamped immediately to avoid exsanguination into the cardiopulmonary bypass reservoir. Only after cessation of arterial flow, an aortic clamp (V. Mueller Cosgrove Flex Clamp, CareFusion, Waukegan, IL) is placed across the aorta where it was previously encircled with the polyethylene tube. The sequence of the circulatory arrest steps is critically important to avoid entraining air into the open arch.

Next, the aneurysm is opened proximally from the crossclamp to the intended proximal anastomotic site in the distal aortic arch (Figure 3). Once the arch is cleared of blood, taking care not to suction blood below the orifices of the innominate and left carotid arteries, we directly inspect the arch for dissection and clot. When there is chronic dissection in the arch after proximal repair of a type A dissection, the septum is fenestrated as far proximally as possible. Once the brief arch inspection has occurred and there is no need to expose or resect more proximally, perfusion from the femoral artery is resumed at 1 to $1.5 \mathrm{~L} / \mathrm{min}$ maintaining partial occlusion of the venous line. A flexible suction catheter (Atra Sump, California Medical Laboratories, Inc., Costa Mesa, CA) is placed in the open proximal aorta avoiding the exposure of the orifices of the innominate or left common carotid arteries to air. If 


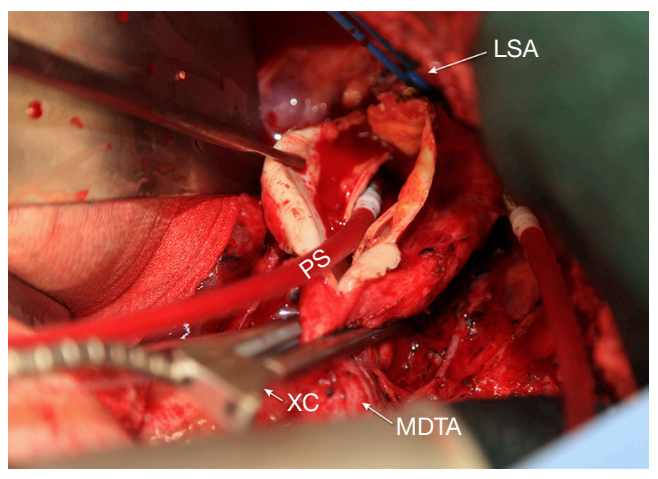

Figure 3 Chronic arch and thoracoabdominal aortic dissection opened between the left subclavian artery and the mid descending aorta during period of DHCA. LSA, left subclavian artery; XC, cross clamp; MDTA, mid descending thoracic aorta; PS, pump sucker.

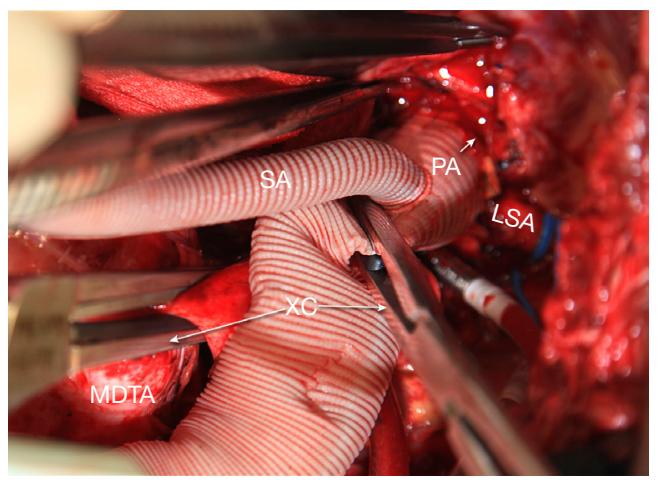

Figure 4 Completed proximal anastomosis with restoration of proximal perfusion via a secondary arterial line. The blue vessel loop is around the left subclavian artery. LSA, left subclavian artery; PA, proximal anastomosis; SA, side arm graft; XC, cross clamp; MDTA, mid descending thoracic aneurysm.

too much blood prevents visualization of the anastomosis during proximal suturing, the venous line can be opened slightly to allow drainage of the heart. It is important to find an area in the distal or transverse arch devoid of thrombus for the proximal anastomosis and at times requires resection of nonaneurysmal aorta.

A $26-30 \mathrm{~mm}$ Dacron graft with a $10 \mathrm{~mm}$ perfusion sidearm (Vascutek Gelweave Graft, Terumo Cardiovascular Corporation, Renfrewshire, Scotland, UK) is sewn to the open proximal aorta using 3-0 polypropylene suture. The side arm is kept within 1-2 $\mathrm{cm}$ of the proximal anastomosis Proximal flow is established via a second arterial line with a separate roller head at $1 \mathrm{~L} / \mathrm{min}$ through the perfusion sidearm. After proximal anastomotic hemostasis is inspected and secured, flow is once again temporarily discontinued proximally and blood is aspirated from the graft just proximal to the anastomosis. The anastomosis is dried on the outside and sealed with a biological adhesive (BioGlue, Cryolife, Inc, Alpharetta, GA). Taking a few extra seconds to seal the anastomosis properly is invaluable especially when multiple anastomoses are anticipated. A cross-clamp is placed immediately distal to the perfusion sidearm and arterial flow is resumed at $1 \mathrm{~L} / \mathrm{min}$ or more to maintain a right radial arterial pressure of $40 \mathrm{mmHg}$ or higher (Figure 4). Left ventricular decompression is resumed at this time. Any back bleeding from intercostal arteries proximal to the cross-clamp are ligated. Ligating these intercostal arteries minimizes steal from spinal cord blood flow.

The patient is then placed in reverse Trendelenburg position and the femoral arterial perfusion is stopped while continuing proximal perfusion with the venous line partially occluded. The cross-clamp at mid descending thoracic aorta is removed and the remainder of the aneurysm is opened. Patent intercostal arteries in the lower descending thoracic aorta are identified and incorporated into the distal anastomosis and spared on the native remaining aorta. If such an anastomosis can complete the repair, the arterial blood is warmed to 37 degrees Centigrade during the suturing of this distal anastomosis.

During the warming phase, after 10 minutes of proximal blood flow at 30 degrees Centigrade or higher, first attempts are made to defibrillate the heart. Lidocaine (100 $\mathrm{mg})$ and magnesium sulfate $(1 \mathrm{~g})$ are administered directly into the cardiopulmonary bypass circuit often help to maintain sinus rhythm once the heart has been defibrillated. Once the ventricles begin to contract, the right lung is ventilated to avoid North-South Syndrome. At this stage with venous flow restricted and right heart partially filled, right lung ventilation may prevent the heart from ejecting deoxygenated blood into the coronary arteries and cerebral circulation.

Once the distal anastomosis is completed and sealed with a biological adhesive, the graft is carefully de-aired. The patient should be switched to Trendelenburg position during de-airing as air can be trapped in the graft. The cross-clamp distal to the side arm is removed. Perfusion is increased to patient's calculated pump flow and venous line drainage is unrestricted. Meticulous surgical hemostasis is obtained and is greatly simplified if there is proper sealing 


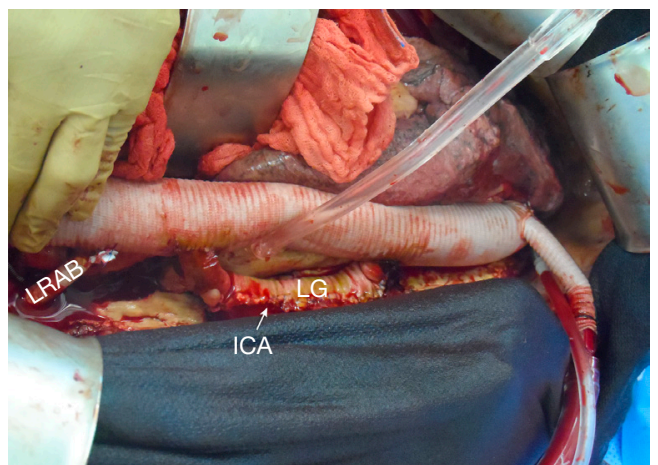

Figure $510 \mathrm{~mm}$ Looped Dacron graft to an intercostal artery pedicle. LG, loop graft; ICA, intercostal artery patch; LRAB, left renal artery bypass graft.

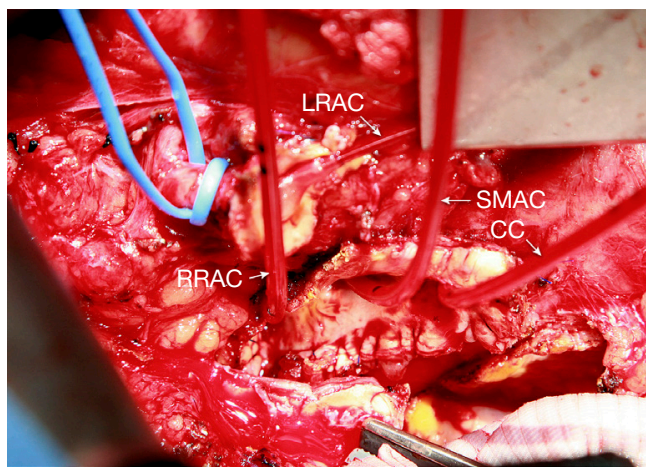

Figure 6 Visceral perfusion catheters placed in the branches of the abdominal aorta. LRAC, left renal artery catheter; SMAC, superior mesenteric artery catheter; CC, celiac artery catheter; RRAC, right renal artery catheter.

of anastomoses. The femoral arterial cannula is removed during warming, perfusing solely through the side arm graft, allowing unimpeded perfusion of the left leg.

Once normothermia and hemostasis is achieved, the left ventricular decompression is removed. The site is repaired while on cardiopulmonary bypass. This repair can be challenging especially if significant aortic insufficiency exists. In this case, dropping cardiopulmonary bypass flow to $1 \mathrm{~L} / \mathrm{min}$ with the venous line unrestricted facilitates this repair.

Weaning from cardiopulmonary bypass frequently requires inotropic support (norepinephrine or epinephrine) in the first few hours to maintain a mean arterial pressure of 70-90 mmHg. Transesophageal echocardiography is useful in assessing ventricular function and volume status.
The venous line is removed and femoral vein repaired taking care not to narrow the cannulation site. The transverse venotomy is closed with 5-0 polypropylene traction sutures placed in opposite corners of the venotomy, and the venotomy is closed with a separate running 5-0 polypropylene suture.

\section{Thoracoabdominal aneurysm repair}

The thoracoabdominal aneurysms are repaired using the same technique as for descending thoracic aneurysm repair proximally. After the proximal anastomosis is completed and higher intercostal arteries ligated, the repair proceeds as follows.

The femoral arterial flow is stopped, the mid descending aortic cross-clamp is removed and remainder of the aneurysm is opened to the aortic bifurcation if necessary. Care is taken not injure the left renal artery, which lies 1 to $2 \mathrm{~cm}$ caudal to the median arcuate ligament.

By necessity, spinal cord and visceral blood flow is reduced during this portion of the operation until the repair has been completed. Therefore, to minimize the ischemic insult to the spinal cord and abdominal viscera, the perfusion circuit remains cold. Attempts are made to keep adequate cold perfusion to one of 3 sources of collateral blood flow to the spinal cord (via the vertebral arteries, segmental intercostal arteries, or internal iliac arteries). Usually at this point during the operation, only proximal perfusion has been resumed and antegrade flow to the vertebral arteries is the only significant perfusion of the spinal cord. Maintaining mean arterial pressure of 50-60 $\mathrm{mmHg}$ with increased proximal arterial flow can achieve adequate spinal cord perfusion when the other sources of spinal cord oxygen delivery are temporarily stopped. Patent intercostal arteries, usually 3-4 pairs, in the region of T8-L1 are revascularized as an island using a $10-\mathrm{mm}$ looped Dacron graft (Figure 5) (Vascutek Gelsoft Straight Graft, Terumo Cardiovascular Corporation, Renfrewshire, Scotland, UK). Only after resuming segmental arterial blood flow to the lower spinal cord, is the patient's arterial blood warmed to 20 degrees Centigrade.

The four visceral branches are perfused at low flow (100-200 $\mathrm{mL} / \mathrm{min}$ ) with 15 -degree Centigrade blood via 9-French balloon perfusion catheters (9F Pruitt Irrigation Occlusion Catheters, LeMaitre Vascular, Inc., Burlington, MA; Figure 6). Study of the preoperative CTA will help identify the origins of the visceral branches which can come off the false lumen or both lumens. These perfusion 


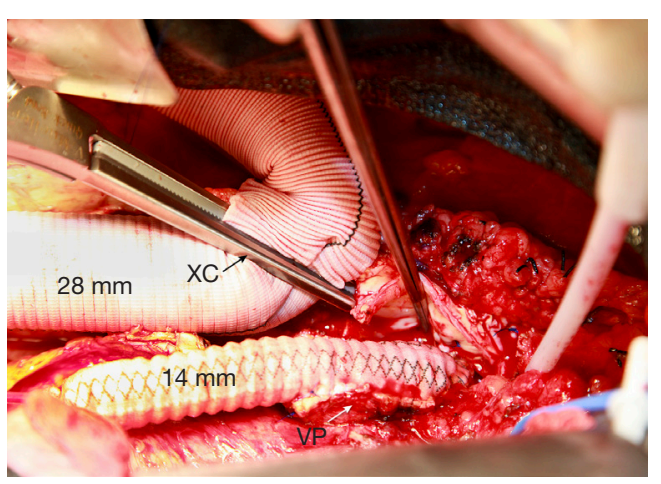

Figure $714 \mathrm{~mm}$ Dacron graft anastomosis to the celiac, superior mesenteric and right renal artery patch. The cross clamp is placed distal to intercostal artery loop graft. VP, visceral patch including celiac, superior mesenteric and right renal arteries; XC, ross clamp.

catheters are placed after opening the lower portion of the aneurysm and prior to removal of thrombus which can be present in the false lumen. Visceral arteries are reattached by separate grafts (a Coselli graft can be useful especially in patient with multiple ostial stenosis or connective tissue disorder, Vascutek, Terumo Cardiovascular Corporation, Renfrewshire, Scotland, UK) or as an island or button. Occasionally, an island of celiac, superior mesenteric and right renal arteries is sutured to a separate $14-\mathrm{mm}$ Dacron graft (Vascutek Gelsoft Straight Graft, Terumo Cardiovascular Corporation, Renfrewshire, Scotland, UK; Figure 7) which in turn is connected to the main thoracoabdominal graft proximally. Flow is re-established to these visceral arteries and the proximal clamp is moved below. At this point, proximal cardiopulmonary bypass flow is increased to 2.5 to $4 \mathrm{~L} / \mathrm{min}$ and the patient is warmed to 30 degrees Centigrade. At 30 degrees if multiple lower intercostals were not reimplanted due to chronic occlusion, the radial arterial pressure is maintained at $80 \mathrm{mmHg}$ or higher since the vertebral arteries may be the primary source of spinal cord blood flow.

The left renal artery is attached either as with an interposition 6-8 mm PTFE graft (Vascutek SEALPTFE Wrap Graft, Terumo Cardiovascular Corporation, Renfrewshire, Scotland, UK) or using an $8 \mathrm{~mm}$ limb of the Coselli multibranched graft (Figures 8). The left renal connection can be easily misaligned and should be inspected once the left kidney is placed back in its anatomical position. Once this anastomosis is complete, the patient is warmed to 37 degrees Centigrade.

The inferior mesenteric artery, if greater than 2 to $3 \mathrm{~mm}$ in diameter and or is associated with chronic occlusion of either the celiac or superior mesenteric artery should be considered for reimplantation. If an inferior mesenteric artery is ligated and diarrheal stool occurs in the first 6 hours postoperatively, one should consider emergent return to the OR and revascularizing the inferior mesenteric artery if possible. Major morbidity and mortality can occur from routine ligation of this artery.

The distal anastomosis is completed at the aortic bifurcation and the thoracoabdominal graft is meticulously de-aired. In the setting of chronic dissection and aneurysm of the iliac artery, an aorto-biiliac graft (Vascutek Gelseal Bifurcate Graft, Terumo Cardiovascular Corporation, Renfrewshire, Scotland, UK) is often required for appropriate distal reconstruction. Typical reconstructions of a Crawford extent II thoracoabdominal aneurysm are shown in Figure 9. The patient is fully rewarmed, the left ventricular decompression catheter removed as outlined above and the patient separated from cardiopulmonary bypass. Protamine is administered and surgical hemostasis is obtained.

An intercostal nerve block is performed on all patients. The diaphragm is closed with a running \#1 polypropylene suture. The abdominal wall musculature is closed in one or two layers with \#1 polydiaxanone suture. Large braided \#5 Ethibond paracostal sutures are placed. The cut ends of the costochondral margin should meet as the ribs are reapproximated and should not overlap to avoid chronic nonunion of the costal margin. Plating of a divided rib can enhance thoracic wall stability and facilitate proper bone healing.

\section{Intraoperative monitoring}

As mentioned above, proximal perfusion to maintain a radial arterial pressure of $40 \mathrm{mmHg}$ or higher during hypothermia should be the goal during the procedure. Increasing cardiopulmonary bypass flow and radial arterial pressure stepwise during warming is essential as mentioned above especially if intercostals were not reimplanted. During warming and once weaned off cardiopulmonary bypass, mean arterial pressures should be maintained between $70-90 \mathrm{mmHg}$ usually requiring low dose vasopressor agents (norepinephrine). Cerebral saturations are usually supranormal during the cooling phase and fall appropriately during the period of circulatory arrest to the brain. The cerebral oximetry values typically return to baseline during 

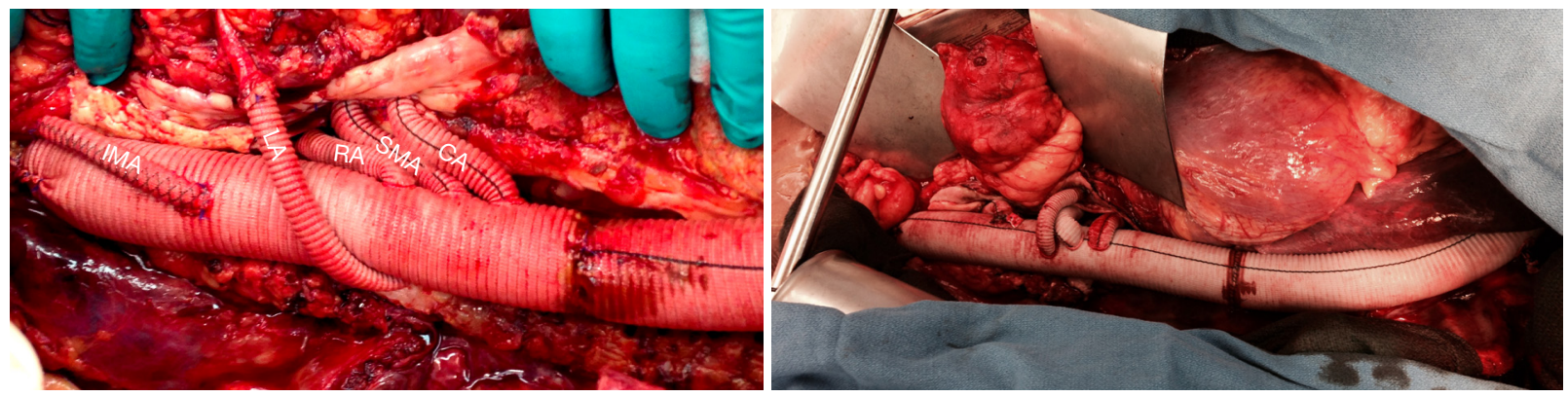

Figure 8 Coselli graft to the visceral arteries and separate bypass to the inferior mesenteric artery. CA-celiac artery; SMA, superior mesenteric artery; RA, right renal artery; LA, left renal artery; IMA, inferior mesenteric artery.
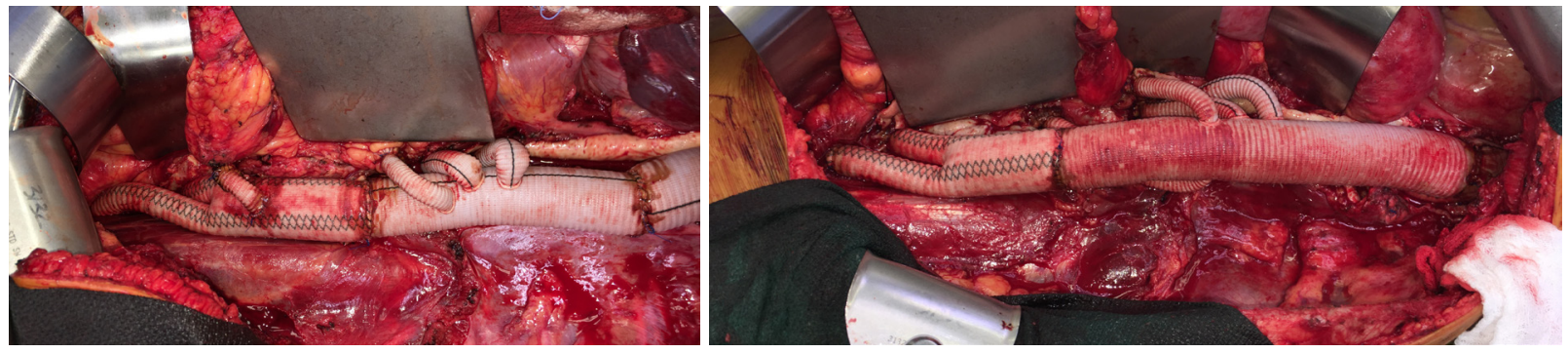

Figure 9 Completed Crawford Extent II thoracoabdominal aneurysm repairs with aorto-biiliac grafts for iliac aneurysms.

rewarming. Motor evoked potentials are assessed once fully rewarmed and separated from cardiopulmonary bypass. The neurological monitoring is unreliable during the period of hypothermia and cardiopulmonary bypass. If the MEPs are not present in the bilateral lower extremities, a subarachnoid drain (Codman Lumbar Drainage Kit II, Johnson \& Johnson Professional, Inc., Rynham, MA) is inserted in the operating room immediately after wound closure and correction of coagulopathy if not already in place. The lumbar drain is opened to remove $10-20 \mathrm{~mL}$ of cerebrospinal fluid per hour and target an intraspinal pressure of less than $10 \mathrm{cmH}_{2} \mathrm{O}$ (18). When MEPs are not present in the lower extremities, the mean arterial pressure is raised to a target of $90-110 \mathrm{mmHg}(19)$. Urine output should be in the range of $50-100 \mathrm{~mL}$ every $30 \mathrm{~min}$ during closing. Sufficient urine output should be observed without additional diuretic stimulus and if it is not observed, renal artery positioning and/or bypasses should be inspected for surgically addressable problems, including kinking or occlusion.

\section{Postoperative management}

Postoperatively, mean arterial pressure is maintained between 70-90 $\mathrm{mmHg}$ for the first 2 to 3 days or longer. If signs of spinal cord ischemia exist, the mean arterial pressure is maintained between $90-110 \mathrm{mmHg}$. If a spinal drain is placed, intraspinal fluid pressure is maintained at $10 \mathrm{cmH}_{2} \mathrm{O}$ or less and oxygen delivery, cardiac output and hemodynamics are optimized $(18,19)$. Postoperative hemoglobin levels should be maintained above $7 \mathrm{mg} / \mathrm{dL}$. If spinal cord ischemia is present, hemoglobin levels are increased to $10 \mathrm{mg} / \mathrm{dL}$. Pulmonary artery catheters are removed when no longer needed for hemodynamic adjustment. Extubation is attempted in the first 24 hours. However, factors other than gas exchange must be considered such as central neurologic deficits, temporary neurologic deficit, left lung contusion, large thoracoabdominal incisions and paraplegia are major indicators for a more gradual weaning process. Renal function is closely monitored as the risk of acute kidney injury can be $5-10 \%$ (10-12). Diuretics are administered for fluid overload once hemodynamic and renal parameters return to normal.

Early mobilization after extubation facilitates pulmonary hygiene and quicker recovery.

A video presentation is provided (Video 1) to highlight important aspects of open thoracoabdominal aneurysm 


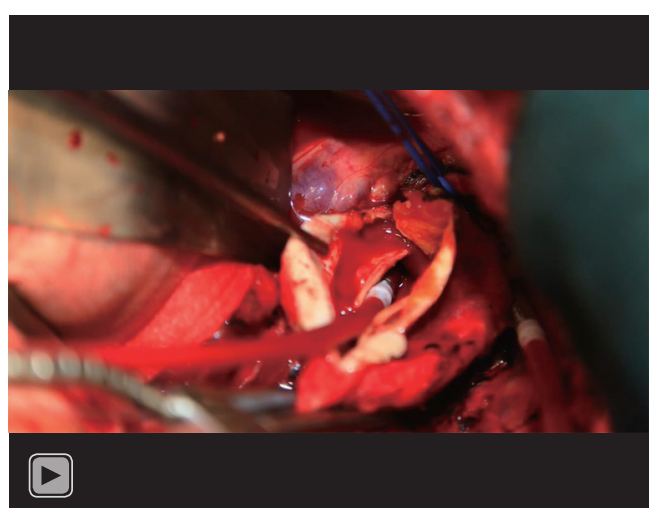

Video 1 A video-graphic presentation of open repair of thoracoabdominal aortic aneurysms using deep hypothermia and circulatory arrest.

repair using our preferred technique of deep hypothermia and circulatory arrest.

\section{Conclusions}

Repair of descending and thoracoabdominal aneurysms in the setting of chronic aortic dissection with open surgery is a high-risk operation with many potential life-altering complications including renal failure, paralysis and stroke. Morbidity and mortality from the procedure is related to the magnitude of the repair with the most extensive repairs, Crawford extents II and III, having the highest complication rates $(20,21)$. Deep hypothermia as a perfusion technique in thoracoabdominal aneurysm repair has been used routinely by Kouchoukos and our group to mitigate end organ ischemia during the repair thereby improving outcomes (10-12,22-24). Although the technique is complex, operative outcomes are excellent when used on a routine basis. The best operative outcomes appear to be in patients with chronic distal aortic dissection with thoracoabdominal aneurysm $(10,12)$.

\section{Acknowledgments}

Funding: None.

\section{Footnote}

Provenance and Peer Review: This article was commissioned by the Guest Editors (Ibrahim Sultan and George Arnaoutakis) for the series "Advancement in the Surgical
Treatment of Aortic Dissection" published in Fournal of Visualized Surgery. The article has undergone external peer review.

Conflicts of Interest: All authors have completed the ICMJE uniform disclosure form (available at https://jovs. amegroups.com/article/view/10.21037/jovs-20-99/coif). The series "Advancement in the Surgical Treatment of Aortic Dissection" was commissioned by the editorial office without any funding or sponsorship. JWF reports personal fees from CryoLife, Inc., outside the submitted work. The authors have no other conflicts of interest to declare.

Ethical Statement: The authors are accountable for all aspects of the work in ensuring that questions related to the accuracy or integrity of any part of the work are appropriately investigated and resolved. All procedures performed in this study were in accordance with the Helsinki Declaration (as revised in 2013). The manuscript is waived from patient informed consent according to the ethics committee or institutional review board.

Open Access Statement: This is an Open Access article distributed in accordance with the Creative Commons Attribution-NonCommercial-NoDerivs 4.0 International License (CC BY-NC-ND 4.0), which permits the noncommercial replication and distribution of the article with the strict proviso that no changes or edits are made and the original work is properly cited (including links to both the formal publication through the relevant DOI and the license). See: https://creativecommons.org/licenses/by-nc-nd/4.0/.

\section{References}

1. Zoli S, Etz CD, Roder F, et al. Long-Term survival after open repair of chronic distal aortic dissection. Ann Thorac Surg 2010;89:1458-66.

2. Pujara AC, Roselli EE, Hernandez AV, et al. Open repair of chronic distal aortic dissection in the endovascular era: Implications for disease management. J Thorac Cardiovasc Surg 2012;144:866-73.

3. Conway AM, Sadek M, Lugo J, et al. Outcomes of open surgical repair for chronic type B aortic dissections. J Vasc Surg 2014;59:1217-23.

4. van Bogerijen GH, Patel HJ, Williams DM, et al. Propensity adjusted analysis of open and endovascular thoracic aortic repair for chronic type B dissection: A twenty-year evaluation. Ann Thorac Surg 2015;99:1260-6. 
5. Estrera AL, Jan A, Sandhu H, et al. Outcomes of open repair for chronic descending thoracic aortic dissection. Ann Thorac Surg 2015;99:786-93; discussion 794.

6. Virtue Registry Investigators. The VIRTUE registry of type B thoracic dissections - study design and early results. Eur J Vasc Endovasc Surg 2011;41:159-66.

7. Parsa CJ, Williams JB, Bhattacharya SD, et al. Midterm results with thoracic endovascular aortic repair for chronic type B aortic dissection with associated aneurysm. J Thorac Cardiovasc Surg 2011;141:322-7.

8. Xu SD, Huang FJ, Yang JF, et al. Early and midterm results 496 of thoracic endovascular aortic repair of chronic type B aortic dissection. J Thorac Cardiovasc Surg 2010;139:1548-53.

9. Leshnower BG, Szeto WY, Pochettino A, et al. Thoracic endografting reduces morbidity and remodels the thoracic aorta in DeBakey III aneurysms. Ann Thorac Surg 2013;95:914-21.

10. Corvera J, Copeland H, Blitzer D, et al. Open Repair of Chronic Thoracic and Thoracoabdominal Aortic Dissection Using Deep Hypothermia and Circulatory Arrest. J Thorac Cardiovasc Surg 2017;154:389-95.

11. Fehrenbacher JW, Siderys H, Terry C, et al. Early and late results of descending thoracic and thoracoabdominal aortic aneurysm open repair with deep hypothermia and circulatory arrest. J Thorac Cardiovasc Surg 2010;140:S154-60.

12. Corvera JS, Fehrenbacher JW. Open repair of chronic aortic dissection using deep hypothermia and circulatory arrest. Ann Thorac Surg 2012;94:78-81; discussion 82-3.

13. Coady MA, Rizzo JA, Hammond GL, et al. What is the appropriate size criterion for resection of thoracic aortic aneurysms? J Thorac Cardiovasc Surg 1997;113:476-91.

14. Davies RR, Goldstein LJ, Coady MA, et al. Yearly rupture or dissection rates for thoracic aortic aneurysms: simple prediction based on size. Ann Thorac Surg 2002;73:17-27.

15. Kim JB, Kim K, Lindsay ME, et al. Risk of rupture or dissection in descending thoracic aneurysms. Circulation

doi: $10.21037 /$ jovs-20-99

Cite this article as: Corvera JS, Hess PJ Jr, Fehrenbacher JW. Open repair of chronic type B aortic dissection. J Vis Surg 2021;7:39.
2015;132:1620-9.

16. Griepp RB, Ergin MA, Galla JD, et al. Looking for the artery of Adamkiewicz: a quest to minimize paraplegia after operations for aneurysms of the descending thoracic and thoracoabdominal aorta. J Thorac Cardiovasc Surg 1996;112:1202-13.

17. Griepp RB, Griepp EB. Spinal cord perfusion and protection during descending thoracic and thoracoabdominal aortic surgery: the collateral network concept. Ann Thorac Surg 2007;83:S865-9.

18. Estrera AL, Sheinbaum R, Miller CC, et al. Cerebrospinal fluid drainage during thoracic aortic repair: safety and current management. Ann Thorac Surg 2009;88:9-15.

19. Estrera AL, Sheinbaum R, Miller CC, et al. Neuromonitor-guided repair of thoracoabdominal aneurysms. J Thorac Cardiovasc Surg 2010;140:S131-5.

20. Svensson LG, Crawford ES, Hess KR, et al. Variables predictive of outcome in 832 patients undergoing repairs of the descending thoracic aorta. Chest 1993;104:1248-53.

21. Crawford ES, Crawford JL, Safi HJ, et al. Thoracoabdominal aortic aneurysm: preoperative and intraoperative factors determining immediate and longterm results of operations in 605 patients. J Vasc Surg 1986;3:389-404.

22. Fehrenbacher JW, Hart DW, Huddleston E, et al. Optimal end-organ protection for thoracic and thoracoabdominal aneurysm repair using deep hypothermic circulatory arrest. Ann Thorac Surg 2007;83:1041-6.

23. Kouchoukos NT, Masetti P, Rokkas CK, et al. Hypothermic cardiopulmonary bypass and circulatory arrest for operations on the descending thoracic and thoracoabdominal aorta. Ann Thorac Surg 2002;74:S1885-7.

24. Kouchoukos NT, Kulik A, Castner CF. Outcomes after thoracoabdominal aneurysm repair using hypothermic circulatory arrest. J Thorac Cardiovasc Surg 2013;145:S139-41. 